\title{
Superoxide Dismutase and Catalase Do Not Affect the Pulmonary Hypertensive Response to Group B Streptococcus in the Lamb
}

\author{
DAVID T. CARPENTER, HEATHER R. LARKIN, AUDREY S. CHANG, ELENA MORRIS, \\ J. TIMOTHY O'NEILL, AND JERRI CURTIS \\ Uniformed Services University of the Health Sciences, Department of Pediatrics, Bethesda, MD \\ 20814-4799 [D.T.C., H.R.L., T.O., J.C.]; Walter Reed Army Medical Center, Department of Clinical \\ Investigation [A.S.C], Department of Biochemistry [E.M.], Washington, D.C. 20307-5001, U.S.A.; \\ National Naval Medical Center, Department of Pediatrics, Bethesda, MD 20889-5600, U.S.A. [J.C.]
}

\begin{abstract}
The purpose of this study was to determine whether treatment with conjugated antioxidant enzymes could attenuate or abolish pulmonary hypertension induced by group B streptococcus (GBS). Lambs, 3-7 d old, were anesthetized and ventilated. Intravascular catheters were placed in the left ventricle, descending aorta, right atrium, and pulmonary artery for continuous monitoring of intravascular pressures. Cardiac output was measured with radiolabeled microspheres. Measurements were obtained at baseline and 15 and 60 min into a 60 -min GBS infusion, and 60 min after GBS was stopped. Blood gas values were held constant and $\mathrm{PaO}_{2}$ was maintained $>100 \mathrm{~mm} \mathrm{Hg}$. The control group received saline vehicle only $(n=6)$, the GBS group received GBS infusion only $(n=9)$, the enzymes (ENZ) group received polyethylene glycol-superoxide dismutase (PEG-SOD) and polyethylene glycol-catalase (PEG-CAT) treatment only ( $n$ $=6$ ), and the ENZ+GBS group received PEG-SOD and PEGCAT then GBS $(n=9)$. Plasma samples were obtained to confirm increased superoxide dismutase and catalase activities in the groups receiving enzymes. Compared with baseline, pulmonary vascular resistance increased by $119 \%$ and $101 \%$ at $15 \mathrm{~min}$ and $87 \%$ and $81 \%$ at $60 \mathrm{~min}$ in the GBS and ENZ+GBS groups, respectively. Sixty minutes after the termination of the GBS infusion, PVR returned to baseline in the GBS group but did not in the ENZ+GBS group. Enzyme infusions resulted in at least a
\end{abstract}

\section{ABSTRACT}

ninefold increase in plasma enzyme activities. As opposed to previously published data from endotoxin models, PEG-CAT and PEG-SOD were ineffective in altering the GBS-induced pulmonary hypertensive response in this model. This suggests that acute administration of antioxidant enzymes may not be effective in ameliorating GBS-induced pulmonary hypertension.

(Pediatr Res 49: 181-188, 2001)

CAT, catalase

\section{Abbreviations:}

CI, cardiac index $(\mathrm{mL} / \mathrm{kg} / \mathrm{min})$

ENZ, enzymes

GBS, group B streptococcus (Streptococcus agalactiae)

MAP, mean systemic arterial pressure $(\mathrm{mm} \mathrm{Hg})$

MPAP, mean pulmonary arterial pressure $(\mathrm{mm} \mathrm{Hg})$

PEG, monomethoxy-polyethylene glycol

PEG-CAT, polyethylene glycol-catalase

PEG-SOD, polyethylene glycol-superoxide dismutase

PVRI, pulmonary vascular resistance index $(\mathrm{mm}$

$\mathrm{Hg} \cdot \mathrm{mL}^{-1} \cdot \mathrm{kg}^{-1} \cdot \mathrm{min}^{-1}$ )

ROS, reactive oxygen species

SOD, superoxide dismutase

SVRI, systemic vascular resistance index $(\mathrm{mm}$

$\mathrm{Hg} \cdot \mathrm{mL}^{-1} \cdot \mathrm{kg}^{-1} \cdot \mathrm{min}^{-1}$ )
Morbidity and mortality remain unacceptably high in newborn infants infected with GBS despite aggressive use

\footnotetext{
Received October 1, 1999; accepted October 13, 2000.
}

Correspondence and reprint requests: Jerri Curtis, CDR, M.C., U.S.N., Uniformed Services University of the Health Sciences, Department of Pediatrics; Room C1066, 4301 Jones Bridge Road, Bethesda, Maryland 20814-4799, U.S.A.

Supported by funds from The Chief, Navy Bureau of Medicine and Surgery, Washington, D.C., Clinical Investigation Program sponsored this study \#B97-077; Uniformed Services University of the Health Sciences \#R086CT; Walter Reed Army Medical Center, Department of Clinical Investigation WU \#6415.

The views expressed in this article are those of the authors and do not reflect the official policy or position of the Department of the Navy, Department of the Army, Department of Defense, nor the U.S. Government. of antibiotics, both intrapartum and immediately following delivery (1). Many infants infected with this organism develop pulmonary hypertension (2), even in the face of blood cultures that have quickly become sterile. In many of these infants, the high morbidity and mortality is a result of the pulmonary hypertension. There are no unique or specific

The research reported herein was conducted according to the principles set forth in the Guide for Care and Use of Laboratory Animals, Institute of Laboratory Animal Resources, National Research Council, HHS, Pub. No. (NIH) 85-23, revised 1985.

Presented in part at the American Academy of Pediatrics Fall Meeting, October 1998; San Francisco, CA, U.S.A. 
therapies available to reverse this abnormal pathophysiologic process once elicited by the group B streptococcal organism. Outcomes have improved over the past decade with the use of extracorporeal membrane oxygenation, inhaled nitric oxide, and more sophisticated high-frequency ventilators. However, these treatment modalities are not specific to this disease process. There is an increasing body of literature suggesting that ROS play a significant role in GBS-induced pulmonary hypertension. Early scavenging of ROS may offer a specific therapeutic treatment regimen in those infants who have this diagnosis. This could dramatically improve their outcomes.

When pulmonary intravascular macrophages engulf bacteria, ROS are produced (3). ROS are essential for bacterial killing, however, they may be a double-edged sword. Several investigators have proposed that ROS are early mediators of pulmonary hypertension (4-6). For example, pulmonary hypertension induced by endotoxin in the sheep is attenuated by catalase, indicating that hydrogen peroxide may participate in the generation of increased pulmonary arterial pressures $(6,7)$. However, other studies have resulted in inconsistent responses $(8-11)$. Olson et al. (12) hypothesized that the short half-life of antioxidant enzymes was responsible for the variability in responses. They attempted to overcome this problem by using antioxidant enzymes that had been conjugated to PEG, which greatly increased the half-life. They used SOD or CAT bound to PEG in a pig model of endotoxin-induced pulmonary hypertension. PEG-SOD alone did not offer any protection, but PEG-CAT significantly blunted the endotoxin-induced pulmonary injury. A combination of the PEG-bound enzymes was not studied.

Other investigators have examined the role of ROS in the development of GBS-induced pulmonary hypertension using dimethylthiourea as a hydroxyl radical scavenger $(4,13-15)$. All have demonstrated significant attenuation of GBS-induced rises in pulmonary artery pressure or pulmonary vascular resistance in the animals pretreated with dimethylthiourea. However, there have been doubts regarding the specificity that dimethylthiourea has for hydroxyl radical scavenging (16). Therefore, the question regarding the role of ROS in the mechanism of GBS-induced pulmonary hypertension remains unanswered.

The aim of the present study was to determine whether early treatment with both conjugated antioxidant enzymes (PEG-SOD and PEG-CAT) could attenuate or abolish pulmonary hypertension induced by GBS. We hypothesized that pulmonary intravascular macrophages are activated by engulfing bacteria, producing an oxidative burst, resulting in the release of ROS into the extracellular space (3). Therefore, the administration of antioxidant enzymes would reduce the ROS concentration and thus the pulmonary vasoconstriction observed with GBS infusions. To test this hypothesis, conjugated antioxidant enzymes (PEG-SOD and PEG-CAT) were intravenously administered before induction of pulmonary hypertension with a continuous infusion of GBS in the newborn lamb.

\section{METHODS}

Animal preparation. Mixed-strain newborn lambs (3-7 d of age) were anesthetized with i.v. sodium pentobarbital (induction dose, $30 \mathrm{mg} / \mathrm{kg}$; maintenance, $5-7 \mathrm{mg} \cdot \mathrm{kg}^{-1} \cdot \mathrm{h}^{-1}$ ). The trachea was intubated and animals were ventilated using a Harvard Model 665 animal volume ventilator (Harvard Apparatus Co., South Natick, MA, U.S.A.). Continuous noninvasive monitoring of end-tidal $\mathrm{Co}_{2}, \mathrm{Fio}_{2}$, and $\mathrm{O}_{2}$ saturation was attained via a BCI Model 9100 multi gas monitor (BCI International, Waukesha, WI, U.S.A.) and Ohmeda 4700 pulse oximeter (Ohmeda Corp., Louisville, CO, U.S.A.). Ventilator settings and supplemental oxygen were adjusted to maintain an

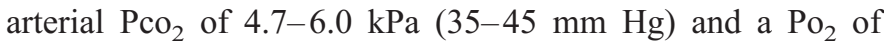
$>13.3 \mathrm{kPa}(>100 \mathrm{~mm} \mathrm{Hg})$. Arterial $\mathrm{pH}$ was maintained between 7.35-7.45, with sodium bicarbonate used as necessary. Rectal temperature was servo-controlled (YSI model 73A, Yellow Springs Instruments, Yellow Springs, OH, U.S.A.) and maintained at $39-40^{\circ} \mathrm{C}$ via a heating blanket and heat lamp wired to a rectal probe. Arterial blood gas values and $\mathrm{pH}$ are reported at the animals' body temperature.

Catheters were placed in the pulmonary artery, left ventricle, right atrium, subclavian artery, femoral artery, and femoral vein through peripheral cutdown sites. Pressure transducers (Statham Db-23 Gould Instrument Systems, Cleveland, OH, U.S.A.) were used for continuous recording of mean and phasic pressures on a polygraphic recorder (Gould eightchannel strip-chart recorder, model 2017-8868-005180, Cleveland, OH, U.S.A.). Left ventricular and pulmonary arterial catheters were placed by observing characteristic pressure waveforms and confirmed in all study animals during the necropsy. Following catheter placement, each animal was paralyzed with $0.10 \mathrm{mg} / \mathrm{kg}$ of pancuronium bromide and anticoagulated with $500 \mathrm{U} / \mathrm{kg}$ of heparin to maintain line patency. There was a 30 -min postoperative stabilization period before the start of the experimental protocol.

Heat-killed GBS preparation. Type III GBS (strain 893) were plated and incubated at $37^{\circ} \mathrm{C}$ overnight on blood agar. Pure colonies were transferred to Todd-Hewett broth and grown in a $37^{\circ} \mathrm{C}$ water bath to $\log$ phase. This bacterial suspension was centrifuged and washed twice with sterile normal saline to remove broth and then heat-killed in a $60^{\circ} \mathrm{C}$ water bath for $1 \mathrm{~h}$. The suspension was then diluted with sterile normal saline to an OD of $0.6\left(1.0 \times 10^{12}\right.$ colony forming units per liter). The final bacterial suspension was cultured to confirm sterility.

Antioxidant enzyme preparation. PEG-SOD (EC 1.15.1.1) and PEG-CAT (EC 1.11.1.6) were purchased from Oxis (Portland, OR, U.S.A.). Both enzymes were tested for the presence of endotoxin using a Limulus amebocyte assay (17) (E-toxate kit; Sigma Chemical Co., St. Louis, MO, U.S.A.). The PEGCAT required endotoxin removal. Endotoxin was removed using a cyclic agarose suspension (Kuttsuclean, Taiyo Fishery Co., Ibaraki, Japan). Greater than $95 \%$ of the catalase activity was retained with undetectable levels of residual endotoxin. SOD and CAT enzyme activities were confirmed spectrophotometrically before administration. One Sigma Chemical Co. unit of CAT activity was defined (by Sigma Chemical Co.) as 
the amount of CAT required to decompose one $1.0 \mathrm{mmol}$ of hydrogen peroxide in $1 \mathrm{~min}$ at $25^{\circ} \mathrm{C}$ and $\mathrm{pH} 7.0$ (18). A unit of SOD activity was defined as the amount of SOD required to produce $50 \%$ inhibition of the rate of reduction of cytomchrome $c$ at $25^{\circ} \mathrm{C}$ and $\mathrm{pH} 7.8$ (19).

Cardiac output. Cardiac output was measured using the radiolabeled microsphere technique (20). Approximately 1.01.5 million radiolabeled microspheres $\left({ }^{46} \mathrm{Sc},{ }^{85} \mathrm{Sr},{ }^{113} \mathrm{Sn}\right.$, or ${ }^{141} \mathrm{Ce}$; New England Nuclear, Boston, MA, U.S.A.) were injected into the left ventricle. Reference samples were withdrawn from the subclavian artery using a Harvard Model 915 withdrawal pump (Harvard Apparatus Co.) at $2 \mathrm{~mL} / \mathrm{min}$ beginning just before and continuing for $1.5 \mathrm{~min}$ after the microsphere injection. The number of microspheres in the injection aliquots and reference samples were determined by counting total radioactivity on a Gamma Trac $2250 \gamma$-counter (Tracor Northern, Austin, TX, U.S.A.). All reference samples contained at least 1000 microspheres providing an accuracy of $\pm 5 \%$ with a confidence interval of $>90 \%$ for flow calculations (21). CI was defined as cardiac output normalized to animal weight $(\mathrm{kg})$. The following equation was used to calculate CI based on microsphere counts:(1)

$$
\begin{aligned}
& \text { Reference withdrawal rate } \\
& \mathrm{CI}=\frac{\times \text { number of microspheres infected }}{\text { Number of microspheres reference sample }} \\
& X \text { body weight }(\mathrm{kg})
\end{aligned}
$$

Using CI measurements and recorded pressures, PVRI and SVRI were calculated from the following equations:

$$
\text { PVRI }=\frac{\text { MPAP }- \text { LVEDP }}{\text { CI }} \quad \text { SVRI }=\frac{\text { MAP }- \text { RAP }}{C I}
$$

where LVEDP is left ventricular end-diastolic pressure and RAP is right atrial pressure.

Protocol. Using a randomized block design, animals were assigned to one of four groups. Group 1, a control group, received saline in place of PEG-enzymes or a GBS infusion ( $n$ $=6$ ). Group 2, GBS group, animals received only an infusion of GBS, no enzymes $(n=9)$. Group 3, ENZ group, received only the antioxidant enzymes but no GBS $(n=6)$. Group 4 , ENZ+GBS group, received the enzymes followed by an infusion of GBS $(n=9)$.

After the postoperative stabilization period, baseline measurements of vascular pressures, cardiac output, $\mathrm{pH}$, and arterial blood gases (Stat Pro 2, Nova Biomedical, Waltham, MA, U.S.A.) were performed. Each animal then received PEG-SOD and PEG-CAT (or saline). The PEG-SOD and PEG-CAT were each diluted to a volume of $5 \mathrm{~mL}$ with saline before administration. Prepared doses were $2000 \mathrm{U} / \mathrm{kg}$ and 15,000 Sigma Chemical Co. U/kg for the PEG-SOD and PEG-CAT, respectively (12). Fifteen minutes elapsed before the GBS (or saline) infusions were begun (time $=0$ ). At time 0 , the GBS and ENZ+GBS groups received a continuous GBS infusion (versus saline in the control and ENZ groups) at $12 \mathrm{~mL} \cdot \mathrm{kg}^{-1} \cdot \mathrm{h}^{-1}$. This infusion was continued for 60 min during which two further cardiac output and arterial blood gas measurements were obtained at $15 \mathrm{~min}$ and $60 \mathrm{~min}$. The GBS or saline infusion was then discontinued and the animal was allowed 60 min to recover. At $120 \mathrm{~min}$, a final cardiac output and arterial blood gas measurement were taken. In addition, blood was obtained at baseline and 15 and $120 \mathrm{~min}$ from half of the animals to assay for plasma SOD and catalase activity. At the conclusion of the experiment, the animal was euthanized with an overdose of i.v. pentobarbital and an autopsy performed to confirm proper positioning of all catheters and closure of the ductus arteriosus.

Data analysis. Data were analyzed using SPSS 8.0 for windows (SPSS Inc., Chicago, IL, U.S.A.). One-way ANOVA was used to compare the baseline variables among the four groups. The means and standard deviations of the primary outcome variable PVRI as well as the other physiologic variables (MPAP, MAP, SVRI, CI) and respiratory variables (arterial $\mathrm{pH}, \mathrm{Pco}_{2}, \mathrm{Po}_{2}$ ) for the four groups were calculated at baseline and 15, 60, and 120 min. Logarithmic transformation was applied to PVRI, due to skewness in the data distribution, before the data analysis. Changes in physiologic variables among the four groups were assessed using GLM (Generalized Linear Model) for repeated measures over the study time points (i.e. 15, 60, and $120 \mathrm{~min}$ ). Using Tukey's HSD test, post hoc multiple pairwise comparisons were performed when there were significant group by time interactions, to determine differences in physiologic changes between groups at specific time points. Wilcoxon signed rank test was used to determine within-group changes over time points. $p$ Values of $<0.05$ were accepted as representing significant differences between all comparisons. Mann-Whitney $U$ test for independent samples and Wilcoxon signed rank test were used to compare plasma enzyme activities at baseline and over time, respectively. $p$ Values of $<0.05$ were accepted as representing statistically significant differences.

This protocol was approved by the institutional review and animal use committees at the National Naval Medical Center, Uniformed Services University of the Health Sciences, and Walter Reed Army Medical Center.

\section{RESULTS}

There were no significant differences among the control, GBS, ENZ, or ENZ+GBS groups in weights $(5.5 \pm 0.8 \mathrm{~kg}, 4.9 \pm 1.1$ $\mathrm{kg}, 5.4 \pm 1.4 \mathrm{~kg}, 4.6 \pm 0.5 \mathrm{~kg})$ or ages $(5.5 \pm 1.4 \mathrm{~d}, 5.0 \pm 1.5 \mathrm{~d}$, $5.2 \pm 1.7 \mathrm{~d}, 6.2 \pm 0.8 \mathrm{~d}$ ) respectively (mean $\pm 1 \mathrm{SD}$ ). All measured physiologic variables (e.g. pressures and resistances) were similar among the groups before the start of the experimental protocol (Table 1, Fig. 1, A, B). Arterial blood gases and $\mathrm{pH}$ were not significantly different among groups at the beginning of the protocol and did not change over time (Table 1).

MPAP was equivalent among groups before the start of the experimental protocol (Fig. 1A). As anticipated, there was no significant change over time in the MPAP measurements in the control or ENZ groups. GBS caused an increase in MPAP in the presence or absence of enzyme treatment. During GBS infusions, at 15 min, both the GBS and ENZ+GBS groups had significant elevations in MPAP compared with the control and ENZ groups ( $p<0.001$ in all comparisons). The percentage increases in MPAP in the GBS and ENZ+GBS groups over 
Table 1. Physiologic variables, arterial blood gas values, and pH by study group

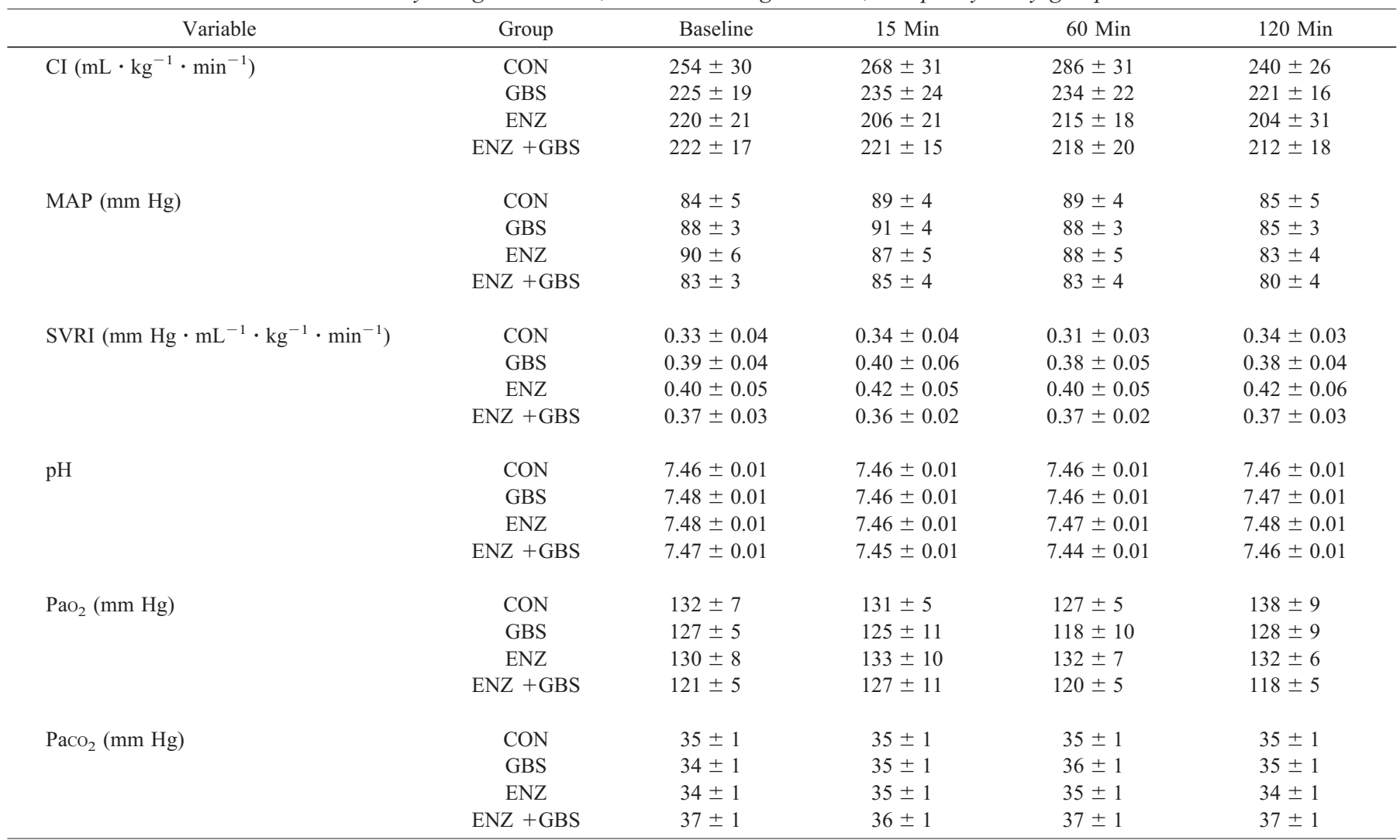

Antioxidant enzymes administered immediately after baseline measurements. Group B streptococcal/saline infusions administered from $0-60$ min.

Groups: CON, control group (no enzymes or GBS; $n=6$ ); GBS group (received only GBS, no enzymes; $n=9$ ); ENZ group (received only antioxidant

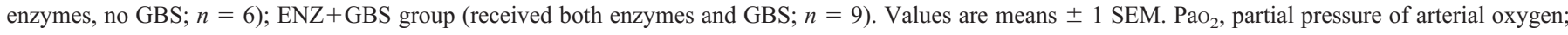
$\mathrm{PaCO}_{2}$, partial pressure of arterial carbon dioxide.

their baseline values were $82 \%$ and $72 \%$, respectively. By 60 min the MPAP in these two groups decreased slightly but continued to be significantly elevated by $62 \%$ and $54 \%$, respectively, and significantly higher than the control group $(p=$ 0.002 and $p=0.001$, respectively). Once the GBS infusion was discontinued, MPAP declined in both the GBS and ENZ+GBS groups. By the 120-min time point, the MPAP in the GBS group had essentially returned to its baseline value, whereas that in the ENZ+GBS group remained significantly elevated ( $p=0.01$ versus control group, $p=0.023$ versus GBS group, $p=0.032$ versus ENZ group).

The PVRI values (Fig. 1B) at baseline were similar among groups. The PVRI values in the control and ENZ groups were similar at all time points and did not change significantly from baseline at any time point. At $15 \mathrm{~min}$ the GBS and ENZ+GBS groups experienced similar rises in PVRI of $119 \%$ and $101 \%$ above their baseline values. Compared with the control group, the PVRI values in both the GBS and ENZ+GBS groups were significantly elevated ( $p=0.013$ and $p=0.01$, respectively). By $60 \mathrm{~min}$, as with the MPAP values, there was a slight diminution in PVRI in both the GBS and ENZ+GBS groups, although they remained elevated compared with the control group $(p=0.016$ and $p=0.004$, respectively). After the discontinuation of the GBS infusion, the PVRI elevation for the GBS+ENZ group at $120 \mathrm{~min}$ remained significantly higher than its baseline $(p=0.008)$, and significantly higher than the control group $(p=0.012)$. PVRI in the GBS group had decreased significantly by $120 \mathrm{~min}$, and was not different from the control or ENZ groups ( $p=0.49$ and $p=$ 0.75 , respectively).

There were no significant differences between groups at any time point for systemic arterial pressure, systemic vascular resistance or cardiac indices (Table 1).

CAT activities in animals that did not receive enzymes were at the lower limit of detection (10 Sigma Chemical Co. U/mL) of the Beers-Sizer assay and remained so throughout the experiment. SOD activities at baseline were similar between animals destined to receive and not receive enzymes with a median concentrations of $5.4 \mathrm{U} / \mathrm{mL}$ and $4.2 \mathrm{U} / \mathrm{mL}$, respectively ( $p=0.82$ ). Ninefold increases in both plasma SOD and CAT activity were seen at the 15-min time point in the animals that received enzymes. These levels of SOD and CAT activity were sustained for almost $2 \mathrm{~h}$, with only $0.2 \%$ and $11 \%$ losses at $120 \mathrm{~min}$, respectively (Fig. 2). Superoxide dismutase activity also fell slightly between $15 \mathrm{~min}$ and $120 \mathrm{~min}$ in animals not receiving antioxidant enzymes to a median concentration of $3.5 \mathrm{U} / \mathrm{mL}$ ( $p=0.046$, data not plotted). There were no differences in enzyme activities between the ENZ group and the ENZ+GBS group.

\section{DISCUSSION}

The aim of our study was to determine whether treatment with both conjugated antioxidant enzymes (PEG-SOD and 

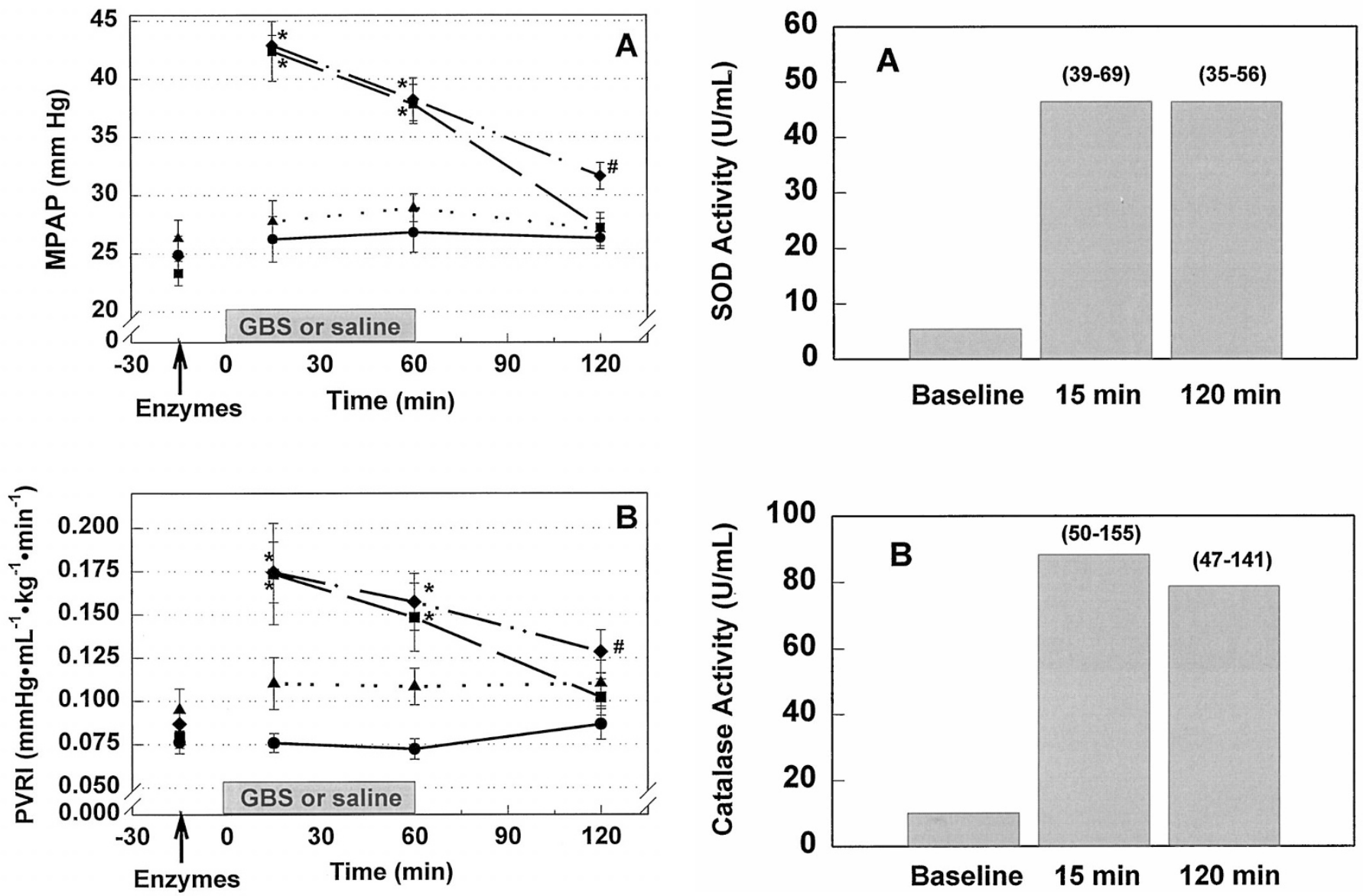

Figure 1. Symbols are means \pm 1 SEM $[\boldsymbol{O}=$ group 1 (control, $n=6)$; $=$ group 2 (GBS only, $n=9$ ); $\boldsymbol{\Delta}=$ group 3 (ENZ only, $n=6$ ); $\diamond$ group 4 (ENZ + GBS, $n=9$ )]. Baseline measurements obtained just before the administration of the antioxidant enzymes. Group B streptococcal/saline infusions started at time $=0$ and continued for $60 \mathrm{~min}$. $(A)$ Effect of the antioxidant enzymes and group B streptococcal infusions on MPAP (mm Hg). GBS infusions produced significant increases in MPAP in both groups treated with GBS at the 15 - and 60 -min time points $\left({ }^{*} p<0.001\right)$. Sixty minutes after the discontinuation of the GBS infusion, the ENZ+GBS group had not fully recovered and still had a significantly elevated MPAP $\left({ }^{\#} p=0.01 v s\right.$ control group, $p=0.023 v s$ GBS group, $p=0.032 v s$ ENZ group). ( $B)$ Effect of the antioxidant enzymes and group B streptococcal infusions on PVRI (mm $\mathrm{Hg} \cdot \mathrm{mL}^{-1} \cdot \mathrm{kg}^{-1} \cdot \mathrm{min}^{-1}$ ). GBS infusions produced significant increases in PVRI in both groups treated with GBS at the 15- and 60-min time points compared with the control group (GBS group: ${ }^{*} p=0.013$ and 0.01 ; ENZ + GBS group: ${ }^{*} p$ $=0.016$ and 0.004 for the respective time points). After the discontinuation of the GBS infusion, the ENZ+GBS group had not fully recovered and still had a significantly elevated PVRI compared with its baseline value $\left({ }^{\#} p<0.008\right)$ and significantly higher than the control group at $120 \mathrm{~min}\left({ }^{\#} p<0.012\right)$.

PEG-CAT) could attenuate or abolish pulmonary hypertension induced by GBS. If this treatment improved outcomes of those animals with GBS-induced pulmonary hypertension then this could possibly offer a new targeted therapeutic treatment plan for sick newborns. Our hypothesis was that GBS bacteremia involved the early and rapid release of superoxide and/or hydrogen peroxide from activated pulmonary intravascular macrophages into the extracellular spaces ultimately resulting in pulmonary hypertension. ROS are produced when pulmonary intravascular macrophages engulf bacteria and become activated (3). If there are high plasma concentrations of antioxidant enzymes bathing these macrophages, then it would be

Figure 2. Plasma antioxidant enzyme levels. Graphs represent median plasma activity concentrations with ranges in parentheses for only those animals that received antioxidant enzymes. $(A)$ Plasma SOD activity. A ninefold increase in SOD activity was measured at the 15 -min time point and was sustained for the duration of the protocol. $(B)$ Plasma CAT activity. The baseline measurement was below the lower limit of the detection of our assay $(<10 \mathrm{U} / \mathrm{mL})$. At least a ninefold increase in CAT activity was measured at the 15 -min time point with only a minimal loss of activity at the 120 -min time point.

possible to inactivate the ROS before their interaction with the surrounding environment. We created ninefold increases in SOD and CAT activities in the plasma in this study. However, this failed to inhibit or attenuate the rise in pulmonary vascular resistance produced by GBS. Nonetheless, important information about the involvement of ROS in GBS-induced pulmonary hypertension was obtained.

The cascade of responses to an acute bacterial challenge have been partially elucidated in several animal models. When GBS bacteria are infused into piglets, they are quickly taken up by pulmonary intravascular macrophages (22). When pulmonary intravascular macrophages engulf bacteria, ROS are produced (3). Considering the close proximity between these macrophages and the pulmonary vascular endothelium, the sudden burst of ROS could interact with the endothelium. This interaction could result in the release of vasoactive substances leading directly or indirectly to the elevation in pulmonary vascular resistance (23-25). Tate et al. (25) demonstrated in a perfused lung model, the generation of $\mathrm{H}_{2} \mathrm{O}_{2}$ produced pulmonary vasoconstriction that could be inhibited by pretreatment with catalase. Martin et al. (24) demonstrated that cultured 
endothelial cells could be damaged by the oxidative burst from activated neutrophils and that this damage was blocked by pretreatment with CAT. These studies clearly demonstrate that ROS, artificially generated, can produce significant damage to endothelial cells and pulmonary vasoconstriction. Normally functioning, intact pulmonary endothelial cells are crucial to maintain the low resting basal tone of the pulmonary circulation. If ROS damages endothelial cells in vivo, then one may expect pulmonary vascular resistance to increase. Thus, therapy aimed at scavenging these ROS might result in a significant attenuation in the pulmonary hypertensive response to an infusion of GBS. In this animal model of GBS-induced pulmonary hypertension, pretreatment with polyethylene glycolbound SOD and CAT did not inhibit the acute rise in pulmonary vascular resistance despite elevated plasma levels of the antioxidant activities. In fact, pretreatment with the antioxidant enzymes may have delayed the recovery of pulmonary vascular resistance in these animals. The results of the present study are in direct opposition to our original hypothesis.

Previous studies indicate that acutely administered SOD and CAT act by scavenging ROS in extracellular spaces $(6,7,26)$. Data from our study indicate that ROS may not penetrate into extracellular spaces. Our results suggest that ROS do not act directly on smooth muscle but may stimulate the production of other vasoactive substances that cause pulmonary vasoconstriction. These substances could be produced by the pulmonary intravascular macrophage themselves or by the endothelium. The pathway for pulmonary intravascular macrophage generated ROS to elicit the production of vasoactive substances by endothelium may be by direct cell-to-cell communication and not diffusion into the extracellular spaces. The fact that dimethylthiourea, which easily penetrates cells, attenuates GBS-induced pulmonary hypertension supports the conclusion that ROS are produced and are acting intracellularly to increase pulmonary vascular resistance. The direct pulmonary intravascular macrophage to endothelium pathway is possible due to their close proximity via adhesive complexes. Large areas of contact between these macrophages and the underlying vascular endothelium have been described (23), which could facilitate this communication. This pathway of cell-to-cell communication has not been previously considered and deserves further investigation.

The use of SOD and CAT in other studies of ROS involvement in models of pulmonary hypertension have yielded inconsistent results $(6,7,10,12,27)$. Possible reasons for the conflicting results could stem from the different doses of the antioxidants used or the time between administration of the antioxidant enzymes and the actual start of some of the experimental protocols. The antioxidant enzymes SOD and CAT have short half-lives $(<10 \mathrm{~min})$, creating much of the variability. We attempted to overcome some of these possible problems by using PEG-bound antioxidant enzymes. The half-life of SOD and CAT can be greatly increased when these enzymes are covalently attached to the inert polymer PEG. Attachment of PEG to these enzymes significantly decreases the renal clearance thus extending the half-life from minutes to greater than $24 \mathrm{~h}$ (28). The inert nature of PEG also reduces the antigenicity of the native protein and inhibits the hydrolysis of the protease-sensitive CAT while maintaining high activity (29). We clearly demonstrated that just one dose of the PEGbound enzymes produced a ninefold increase in the plasma enzyme activities that remained stable for the duration of the protocol (120 $\mathrm{min})$. We assumed a ninefold increase in activities would be therapeutic.

Possible explanations for our unexpected results could include the dosage of the antioxidant enzymes used in this study. The dosage selected for this study was extrapolated from the literature (12). Olson et al. (12) used a piglet model to evaluate the effect of these enzymes on endotoxin-induced pulmonary hypertension. PEG-CAT $(15,000 \mathrm{U} / \mathrm{kg})$ significantly attenuated the endotoxin-induced rise in pulmonary vascular resistance. However, PEG-SOD (2000 U/kg) did not have any effect on the increase in pulmonary vascular resistance with the endotoxin infusion. Moreover, Olson et al. did not use both antioxidant enzymes in combination to determine whether there was a potentiated benefit. This might be expected due to the biochemical association of the superoxide anion and hydrogen peroxide. Some investigators have found a beneficial effect from the use of SOD. Koyama et al. (27) did demonstrate significant attenuation of endotoxin-induced pulmonary hypertension with human-recombinant SOD (4200 U/kg).

Olson et al. (12) also reported that at higher doses of PEG-CAT $(>20,000 \mathrm{U} / \mathrm{kg})$ there were increases in the baseline pulmonary vascular resistance of the animals. This led the investigators to select a lower dose of PEG-CAT to use before the endotoxin infusions. We elected to use $15,000 \mathrm{U} / \mathrm{kg}$ of PEG-CAT in conjunction with $2000 \mathrm{U} / \mathrm{kg}$ of PEG-SOD. This did appear to slightly, but not significantly, increase the baseline resting tone of the pulmonary vasculature. Therefore, we believed our dosage to be optimal, large enough to cause a ninefold increase in plasma activity with minimal direct effects. The pulmonary artery pressure and pulmonary vascular resistance in the enzyme group were only increased by $10 \%$ and $16 \%$, respectively, above its pretreatment baseline. To account for this increase in baseline vascular tone produced by the PEG-bound enzymes, we analyzed the data adjusting for the baseline measurements within each group. Our conclusions did not change following these adjustments.

As stated above, Olson et al. (12) did not report any reduction in pulmonary hypertension with PEG-SOD. We elected to use PEG-SOD in combination with PEG-CAT in this study due to the reported effects that the superoxide anion can have on nitric oxide. The superoxide anion is generally not considered one of the seriously dangerous reactive oxygen species generated during an oxidative burst. However, there are several studies that incriminate it in the destruction of nitric oxide (30). Nitric oxide is the substance identified as having a significant role in maintaining the low basal resting tone of the pulmonary vascular bed (31). The superoxide anion can combine with nitric oxide leading to the production of the peroxynitrite anion, thereby removing the dilatory action of nitric oxide. In addition, the peroxynitrite anion can induce lipid peroxidation and further decompose to yield the hydroxyl radical (32). The hydroxyl radical can produce significant cell damage. Beckman et al. (33) suggested that SOD could reduce the oxidant injury to endothelial cells by preventing the formation of 
peroxynitrite. Thus, the superoxide anion has several potential avenues that can result in pulmonary vasoconstriction. Our data indicate that if these actions of superoxide are operative in GBS-induced pulmonary hypertension, they are not altered by acute administration of PEG-SOD and therefore might occur intracellularly.

The dosages of antioxidant enzymes used may have disrupted the oxidant-antioxidant balance. There has been a bellshaped dose-response curve described for the efficacy of SOD (34). Nelson et al. (34), using an isolated perfused rabbit heart ischemia/reperfusion model, observed that lipid peroxidation was increased when low and high doses of SOD were used. At moderate doses, SOD effectively decreased the rates of lipid peroxidation. There are several studies indirectly linking lipid peroxidation to significant endothelial cell dysfunction (35, 36). In an in vitro system, Mao et al. (37) demonstrated that increasing amounts of SOD in the presence of $\mathrm{Fe}^{+2}$ results in the production of excess amounts of the extremely toxic hydroxyl radical. Mao et al. speculated that as SOD produces $\mathrm{H}_{2} \mathrm{O}_{2}$, if $\mathrm{Fe}^{+2}$ is present, this results in the production of the highly toxic hydroxyl radical via the Fenton reaction. Production of the hydroxyl radical could worsen cellular injury. This could be why the ENZ+GBS group did not recover by the end of the 120-min observation time point compared with the GBS group. Conversely, once the inciting event (i.e. GBS infusion) was stopped, the prolonged presence of the conjugated antioxidant enzymes in the ENZ+GBS group may have continued to scavenge any ROS present, potentiating lipid peroxidation and subsequent endothelial cell damage.

The above-cited studies may certainly explain the diverse results one observes with SOD. The oxidant load and the amount of antioxidant present will determine whether there is a beneficial or detrimental effect. Striking the perfect balance appears critical.

An alternative explanation for our findings could be that there are other vasoactive mediators that play a more important role in the production of the pulmonary hypertension induced by GBS. Several investigators $(4,38)$ have found elevated levels of vasoactive substances (e.g. thromboxane, prostaglandin $\mathrm{B}_{2}$, tumor necrosis factor- $\alpha$, etc.) when studying GBSinduced pulmonary hypertension. Pulmonary intravascular macrophages do produce these substances as well as many others when activated (39). Several investigators have proposed that ROS, when produced during the activation of the macrophage, stimulate the release of these other vasoactive substances, which in turn ultimately lead to pulmonary hypertension (4-6). The ROS may stay intracellular and thus the elevated extracellular antioxidant levels we produced would have no effect on the elevated intracellular levels of ROS.

If the sudden increase in ROS ultimately leads to the production of other vasoactive substances that are then released by the pulmonary intravascular macrophages, then administering the PEG-antioxidant enzymes several hours versus minutes before the infusion of GBS may have attenuated the elevation of pulmonary vascular resistance in these animals. Beckman et al. (40) demonstrated that polyethylene-bound SOD and CAT become incorporated into the cell membranes of cultured endothelial cells and then become intracellularly located over time. Elevated intracellular antioxidant levels would have the advantage of potentially scavenging the ROS before their activation of other vasoactive substances.

Our findings contrast those of the endotoxin model of pulmonary hypertension (12). The disparity between their results and ours is intriguing considering that GBS and endotoxin models of pulmonary hypertension have many features in common. It is possible that endotoxin and GBS act via different pathways, one (endotoxin) in which the extracellular elaboration of superoxide and/or hydrogen peroxide is a critical step, and the other (GBS) in which it is not. However, this study examined only one treatment regimen. ROS may still play a role in GBS-induced pulmonary hypertension. Different doses of the conjugated antioxidant enzymes and/or extended periods of treatment could influence intracellular enzyme activity and lead to a different outcome. Further exploration of possible therapeutic options with conjugated antioxidant enzymes is still warranted.

\section{REFERENCES}

1. Zangwill KM, Schuchat A, Wenger JD 1992 Group B streptococcal disease in the United States, 1990: report from a multistate active surveillance system. MMWR CDC Surveill Summ 41:25-32

2. Shankaran S, Farooki ZQ, Desai R $1982 \beta$-Hemolytic streptococcal infection appearing as persistent fetal circulation. Am J Dis Child 136:725-727

3. Johnston Jr RB 1978 Oxygen metabolism and the microbicidal activity of macrophages. Federation Proc 37:2759-2764

4. Shook LA, Pauly TH, Marple SL, Horstman SJ, Tai HH, Bowdy BD, Gillespie MN 1990 Group B streptococcus promotes oxygen radical-dependent thromboxane accumulation in young piglets. Pediatr Res 27:349-352

5. Liu F, Orr JA, Wu JY 1994 Prostaglandin $\mathrm{B}_{2}$-induced pulmonary hypertension is mediated by $\mathrm{TxA}_{2} / \mathrm{PGH}_{2}$ receptor stimulation. Am J Physiol 267:L602-L608

6. Seekamp A, Lalonde C, Zhu DG, Demling R 1988 Catalase prevents prostanoid release and lung lipid peroxidation after endotoxemia in sheep. J Appl Physiol $65: 1210-1216$

7. Milligan SA, Hoeffel JM, Goldstein IM, Flick MR 1988 Effect of catalase on endotoxin-induced acute lung injury in unanesthetized sheep. Am Rev Respir Dis 137:420-428

8. Crapo JD, DeLong DM, Sjostrom K, Hasler GR, Drew RT 1977 The failure of aerosolized superoxide dismutase to modify pulmonary oxygen toxicity. Am Rev Respir Dis 115:1027-1033

9. Traber DL, Adams TJ, Sziebert L, Stein M, Traber L 1985 Potentiation of lung vascular response to endotoxin by superoxide dismutase. J Appl Physiol 58:10051009

10. Maunder RJ, Winn RK, Gleisner JM, Hildebrandt J, Harlan JM 1988 Effect of intravenous catalase on the pulmonary vascular response to endotoxemia in goats. J Appl Physiol 64:697-704

11. Huang YT, Nozik ES, Piantadosi CA 1994 Superoxide dismutase potentiates plateletactivating factor-induced injury in perfused lung. Am J Physiol 266:L246-L254

12. Olson NC, Grizzle MK, Anderson DL 1987 Effect of polyethylene glycol-superoxide dismutase and catalase on endotoxemia in pigs. J Appl Physiol 63:1526-1532

13. Shook LA, Pauly TH, Horstman SJ, Marple SL, Gillespie MN 1990 Dimethylthiourea reverses sepsis-induced pulmonary hypertension in piglets. Am J Med Sci 300:29-32

14. Bowdy BD, Marple SL, Pauly TH, Coonrod JD, Gillespie MN 1990 Oxygen radical-dependent bacterial killing and pulmonary hypertension in piglets infected with group B streptococci. Am Rev Respir Dis 141:648-653

15. Pauly TH, Bowdy BD, Haven CA, Barr SB, Gillespie MN 1988 Evidence for hydroxyl radical involvement in group B streptococcus-induced pulmonary hypertension and arterial hypoxemia in young piglets. Pediatr Res 24:735-739

16. Wasil M, Halliwell B, Grootveld M, Moorhouse CP, Hutchison DC, Baum H 1987 The specificity of thiourea, dimethylthiourea and dimethyl sulphoxide as scavengers of hydroxyl radicals. Biochem J 243:867-870

17. Levin J 1987 The limulus amebocyte lysate test: perspectives and problems. In: Watson SW, Levin J, Novitsky TJ (eds) Progress in Clinical and Biological Research: Detection of Bacterial Endotoxins with the Limulus Amebocyte Lysate Test. Alan R. Liss, New York, pp 1-23

18. Beers Jr RF, Sizer IW 1952 A spectrophotometric method for measuring the breakdown of hydrogen peroxide by catalase. J Biol Chem 195:133-140

19. Crapo JD, McCord JM, Fridovich I 1978 Preparation and assay of superoxide dismutases. Methods Enzymol 52:382-393

20. Heymann MA, Payne BD, Hoffman JI, Rudolph AM 1977 Blood flow measurements with radionuclide-labeled particles. Prog Cardiovasc Dis 20:55-79

21. Buckberg GD, Luck JC, Payne DB, Hoffman JIE, Archie JP, Fixler DE 1971 Some sources of error in measuring regional blood flow with radioactive microspheres. J Appl Physiol 31:598-604 
22. Bowdy BD, Aziz SM, Marple SL, Yoneda K, Pauly TH, Coonrod JD, Gillespie MN 1990 Organ-specific disposition of group B streptococci in piglets: evidence for a direct interaction with target cells in the pulmonary circulation. Pediatr Res 27:344-348

23. Warner AE, Brain JD 1990 The cell biology and pathogenic role of pulmonary intravascular macrophages. Am J Physiol 258:L1-L12

24. Martin WJ 1984 Neutrophils kill pulmonary endothelial cells by a hydrogenperoxide-dependent pathway. Am Rev Respir Dis 130:209-213

25. Tate RM, Repine JE 1983 Hydrogen peroxide causes permeability edema and hypertension in isolated salt-perfused rabbit lungs. Chest $83: 48 \mathrm{~S}-50 \mathrm{~S}$

26. Kennedy TP, Rao NV, Hopkins C, Pennington L, Tolley E, Hoidal JR 1989 Role of reactive oxygen species in reperfusion injury of the rabbit lung. $\mathrm{J}$ Clin Invest $83: 1326-1335$

27. Koyama S, Kobayashi T, Kubo K, Sekiguchi M, Ueda G 1992 Recombinant-human superoxide dismutase attenuates endotoxin-induced lung injury in awake sheep. Am Rev Respir Dis 145:1404-1409

28. Pyatak PS, Abuchowski A, Davis FF 1980 Preparation of a polyethylene glycol: superoxide dismutase adduct, and an examination of its blood circulation life and anti-inflammatory activity. Res Commun Chem Pathol Pharmacol 29:113-127

29. Abuchowski A, McCoy JR, Palczuk NC, van Es T, Davis FF 1977 Effect of covalent attachment of polyethylene glycol on immunogenicity and circulating life of bovine liver catalase. J Biol Chem 252:3582-3586

30. Gryglewski RJ, Palmer RM, Moncada S 1986 Superoxide anion is involved in the breakdown of endothelium-derived vascular relaxing factor. Nature 320:454-456

31. Fineman JR, Heymann MA, Soifer SJ $1991 \mathrm{~N}$ omega-nitro-L-arginine attenuates endothelium-dependent pulmonary vasodilation in lambs. Am J Physiol 260:H1299-H1306
32. Radi R, Beckman JS, Bush KM, Freeman BA 1991 Peroxynitrite-induced membrane lipid peroxidation: the cytotoxic potential of superoxide and nitric oxide. Arch Biochem Biophys 288:481-487

33. Beckman JS, Beckman TW, Chen J, Marshall PA, Freeman BA 1990 Apparent hydroxyl radical production by peroxynitrite: implications for endothelial injury from nitric oxide and superoxide. Proc Natl Acad Sci U S A 87:1620-1624

34. Nelson SK, Bose SK, McCord JM 1994 The toxicity of high-dose superoxide dismutase suggests that superoxide can both initiate and terminate lipid peroxidation in the reperfused heart. Free Radic Biol Med 16:195-200

35. Brigham KL 1985 Metabolites of arachidonic acid in experimental lung vascular injury. Federation Proc 44:43-45

36. Demling RH, Lalonde C, Jin L-J, Ryan P, Fox R 1986 Endotoxemia causes increased lung tissue lipid peroxidation in unanesthetized sheep. J Appl Physiol 60:2094-2100

37. Mao GD, Thomas PD, Lopaschuk GD, Poznansky MJ 1993 Superoxide dismutase (SOD)-catalase conjugates: role of hydrogen peroxide and the Fenton reaction in SOD toxicity. J Biol Chem 268:416-420

38. Gibson RL, Truog WE, Henderson Jr WR, Redding GJ 1992 Group B streptococcal sepsis in piglets: effect of combined pentoxifylline and indomethacin pretreatment. Pediatr Res 31:222-227

39. Nathan CF 1987 Secretory products of macrophages. J Clin Invest 79:319-326

40. Beckman JS, Minor Jr RL, White CW, Repine JE, Rosen GM, Freeman BA 1988 Superoxide dismutase and catalase conjugated to polyethylene glycol increases endothelial enzyme activity and oxidant resistance. J Biol Chem 263:6884-6892 